\title{
CRITĖRES MORPHOLOGIQUES POUR L'IDENTIFICATION DES DEUX FORMES (RIVIĖRE ET MER) DE TRUITE (SALMO TRUTTA) PRÉSENTES SUR UN MÊME BASSIN.
}

\author{
J.L. BAGLINIÈRE (1), D. OMBREDANE $(1,2)$ et F. MARCHAND (3)
}

(1) UMR INRA-ENSAR, Ecobiologie et Qualité des Hydrosystèmes Continentaux, 65 rue de St Brieuc, 35042 Rennes Cedex, France, E-mail : baglini@roazhon.inra.fr

(2) ENSAR, Laboratoire Ecologie et Sciences phytosanitaires, 65 rue de St Brieuc, 35042 Rennes Cedex, France.

(3) INRA, Unité Expérimentale d'Ecologie et d'Ecotoxicocologie Aquatique, 65 rue de St Brieuc 35042 Rennes Cedex, France.

\section{RÉSUMÉ}

La truite (Salmo trutta) présente deux formes écologiques « rivière " et " mer » qui cohabitent sur de nombreux fleuves du littoral Manche-Atlantique. Ces deux formes sont considérées comme formant un seul stock en raison de la production d'une forme à partir de l'autre et de leurs possibilités de croisement lorsqu'elles sont en sympatrie sur les zones de reproduction. L'étude réalisée montre l'existence de critères morphologiques fiables permettant une identification de ces deux formes au cours de leur cycle biologique. Chez le juvénile, la distinction est impossible au stade résident, mais est possible au stade migrant à partir du taux d'argenture qui est élevé pour la forme marine (groupe des smolts). Chez l'adulte, la distinction est rendue facile par l'analyse des critères de taille et de couleur mais doit être vérifiée par une étude scalimétrique. L'identification de ces deux formes permet alors de mesurer leur niveau d'abondance et d'analyser leur évolution sur le long terme sur un même bassin.

Mots-clés : stock, forme écologique, identification, truite, biométrie, morphologie.

\section{MORPHOLOGICAL CRITERIA FOR IDENTIFICATION \\ OF TWO FORMS (RIVER, SEA) OF BROWN TROUT (SALMO TRUTTA) PRESENT IN THE SAME RIVER.}

\section{ABSTRACT}

The brown trout (Salmo trutta) is living under two forms, river and sea, in many rivers along the Atlantic and Channel coasts. These two forms are considered as coming from the same stock because each form can produces the other one and they can interbreed between them in sympatric spawning area. The present study showed there 
existed some morphological reliable criteria for the identification between the two forms during their biological cycle. In resident and migrating juvenile fish, the distinction was only possible at the migrating stage because of the higher silvering rate in sea form (smolt group) than in river form (parr group). In adult fish, the distinction is possible when size and color criteria were used but it had to check by a scale-reading method. Distinction between the two forms allows to measure the abundance level and their long-term evolution in the same river.

Key-words : stock, ecological form, identification, brown trout, biometry, morphology.

\section{INTRODUCTION}

La truite commune présente une grande plasticité écologique de par son caractère migratoire optionnel et une forte capacité adaptative de par sa colonisation de milieux variès (BAGLINIÈRE, 1991). Ces deux caractéristiques se traduisent chez cette espèce par la présence de trois formes écologiques : résidente (dulçaquicole), migratrice marine et migratrice lacustre. Les deux premières formes qui cohabitent dans de très nombreux cours d'eau du littoral Manche-Atlantique (BAGLINIÈRE, ibidem) présentent quelques différences notables dans leur stratégie démographique (EUZENAT et al., 1991 ; MAISSE et BAGLINIËRE, 1991) : grossissement des sub-adultes en eau douce, faible amplitude de migration, et petite taille des géniteurs avec un rapport des sexes en faveur des mâles chez la forme rivière contre grossissement en mer des sub-adultes, grande amplitude de migration et taille élevée des géniteurs avec un rapport des sexes en faveur des femelles chez la forme marine. Par ailleurs, en conditions de cohabitation, il existe des interactions biologiques qui complexifient la situation.

Les juvéniles des deux formes migrent vers leurs zones d'engraissement à la même période soit au printemps. Ils présentent divers phénotypes dont la reconnaissance visuelle n'est pas précise entraînant quelques difficultés pour leur rattachement à une forme (marine ou rivière) ; cette migration chez la forme résidente correspond au mécanisme de recrutement en truite d'un cours d'eau (MAISSE et BAGLINIĖRE, ibidem). Chez la forme marine, cette migration implique un phénomène de smoltification mais qui ne semble pas aussi marqué que chez le saumon atlantique (TANGUY et al., 1994).

$\|$ existe un chevauchement plus ou moins important des zones de reproduction auquel s'ajoute l'interfécondité entre les deux formes, amplifiée dans certains cas par une absence de barrières éthologiques (taille identique des géniteurs des deux formes).

Aucune différenciation génétique claire n'a pu être décelée entre individus migrateurs et sédentaires originaires d'un même cours d'eau dans cette région géographique (GUYOMARD, 1991 ; BAGLINIĖRE et al., sous presse). En revanche, l'existence de barrières reproductives partielles entre populations génétiquement très proches a pu être mise en évidence dans certains lacs (NÄSLUND, 1993).

Le caractère migratoire marin est partiellement héritable et le déterminisme de migration y compris en mer est sous linfluence du paramètre croissance (OMBREDANE et al., 1998a). Ces deux facteurs étant dépendants de la ou des souches de truite présentes sur le bassin. Notamment, cette dépendance fait que la smoltification peut être induite par la seule action des facteurs externes chez certaines souches de poissons domestiques en milieu naturel. De cela, il résulte que chacune des deux formes peut produire des juvéniles de l'autre forme (OMBREDANE, données non publiées). 
Ces interactions liées à la grande plasticité écologique de la truite sont parfaitement illustrées par l'évolution d'une population de truites de souche domestique qui, introduite aux lles Kerguelen, a donné naissance à des populations de truites lacustre et marine bien établies (DAVAINE et BEALL, 1997). De plus, au vu de ces interactions, il semble que l'on puisse alors apparenter les populations de truites de mer et de rivière aux deux composantes d'un type de métapopulation même si actuellement les deux formes présentes sur un même bassin n'ont pas été caractérisées génétiquement et que leurs flux géniques ne sont pas connus.

Indépendamment, de leurs fortes interactions biologiques, il n'en reste pas moins qu'il est nécessaire de pouvoir distinguer les deux formes présentes sur un même bassin. En effet, le suivi comparatif à long terme de leur population revêt un grand intérêt qui se place à la fois dans le domaine scientifique (Biologie évolutive et Biologie de la conservation) mais également dans celui de la gestion du stock. Cette évolution doit être analysée au travers de l'impact des activités humaines (dégradation de la qualité générale du milieu eau douce, modifications du milieu océanique, conditions différentes d'exploitation des deux formes).

Ainsi, ce travail a pour objectif de rechercher l'existence de critères externes suffisamment fiables permettant de reconnaître les deux formes à certains stades biologiques clés. Ce travail est réalisé à partir de l'analyse de données récoltées à la fois sur les adultes et les juvéniles des populations de truite de quelques cours d'eau français possédant les deux formes. Par ailleurs, il s'inscrit dans le cadre d'une étude plus spécifique concernant la caractérisation morphologique du stade smolt chez la truite.

\section{MATÉRIEL ET MÉTHODES}

\section{Données récoltées}

Au stade adulte, les données proviennent de poissons capturés par piégeage à la montée sur plusieurs grands cours d'eau français (la Garonne, la Dordogne et le Rhin) et sur l'Oir (affluent de la Sélune, Basse-Normandie) où les populations de salmonidés sont suivies depuis plus de 15 ans dans le cadre d'un programme d'étude INRA-CSP concernant la dynamique de population (BAGLINIĖRE et al., 1997).

Au stade juvénile, les données sont issues de poissons capturés soit par pêche électrique réalisée en automne sur l'Oir (poisson résident) soit par piégeage lors de la migration printanière à la fois sur l'Oir et sur le Douet de la Taille (affluent de la Touques, Basse-Normandie).

\section{Critères d'identification utilisés}

Chez l'adulte, les critères retenus sont les suivants : taille (longueur totale ou à la fourche) et livrée, caractérisée par l'argenture et le type de ponctuation. L'appartenance de ces poissons à l'une des deux formes effectuée à partir de ces deux critères a ensuite été vérifiée par scalimétrie en utilisant les normes d'interprétation établies par RICHARD et BAGLINIÉRE (1990) pour la truite de mer.

Ces mêmes critères ont été utilisés à la fois chez les juvéniles résidents et migrants. Néanmoins, chez ces derniers, une analyse morphologique plus approfondie a été réalisée. Par la suite, une analyse du taux d'argenture a été effectuée en liaison avec les modifications physiologiques des individus. En effet, l'augmentation de couleur argentée est en relation étroite avec celle de l'activité de l'ATPase $\mathrm{Na}+\mathrm{K}+$ branchiale qui est un indicateur performant de la capacité du juvénile à passer en mer donc de la smoltification (TANGUY, 1993). Ainsi, ces juvéniles migrants ont d'abord été répartis selon les quatre types morphologiques établis à partir de certaines caractéristiques externes par RICHARD 
(données non publiés) et TANGUY (1993) (Tableau 1). Puis pour chaque phénotype, le coefficient moyen de condition $\mathrm{K}$ a été calculé par la formule $\mathrm{K}=10^{5} \times \mathrm{P} / \mathrm{Lf}^{3}$ avec $\mathrm{P}$ en grammes et Lf en millimètres. Enfin, deux mesures ont été effectuées sur tous les poissons quel que soit le phénotype. Le niveau d'activité de la $\mathrm{Na}+\mathrm{K}+\mathrm{ATPase}$ branchiale a été dosé selon la méthodologie proposée par TANGUY (1993) et TANGUY et al. (1994). Le degré d'argenture à été quantifié à l'aide de mesures colorimétriques faites sur différentes parties du corps (ventre en dessous de la ligne latérale, nageoire pelvienne, opercule et dos) et en tenant compte des résultats acquis chez le saumon atlantique. Les mesures ont été effectuées dans l'espace couleur $L^{*}{ }^{*} b$ où $L$ est la clarté, a la chromaticité du rouge au vert et b la chromaticité du bleu au jaune (OMBREDANE, 2000).

Les données ont été comparées à l'aide de tests statistiques classiques non paramétriques (Wilcoxon-Mann-Whitney et Kruskal-Wallis).

\section{Tableau I}

Caractéristiques externes des différents types morphologiques de truite (RICHARD, com. pers. ; TANGUY, 1993).

\section{Table I}

External characteristics of various of morphological types young brown trout (RICHARD, com. pers. ; TANGUY, 1993).

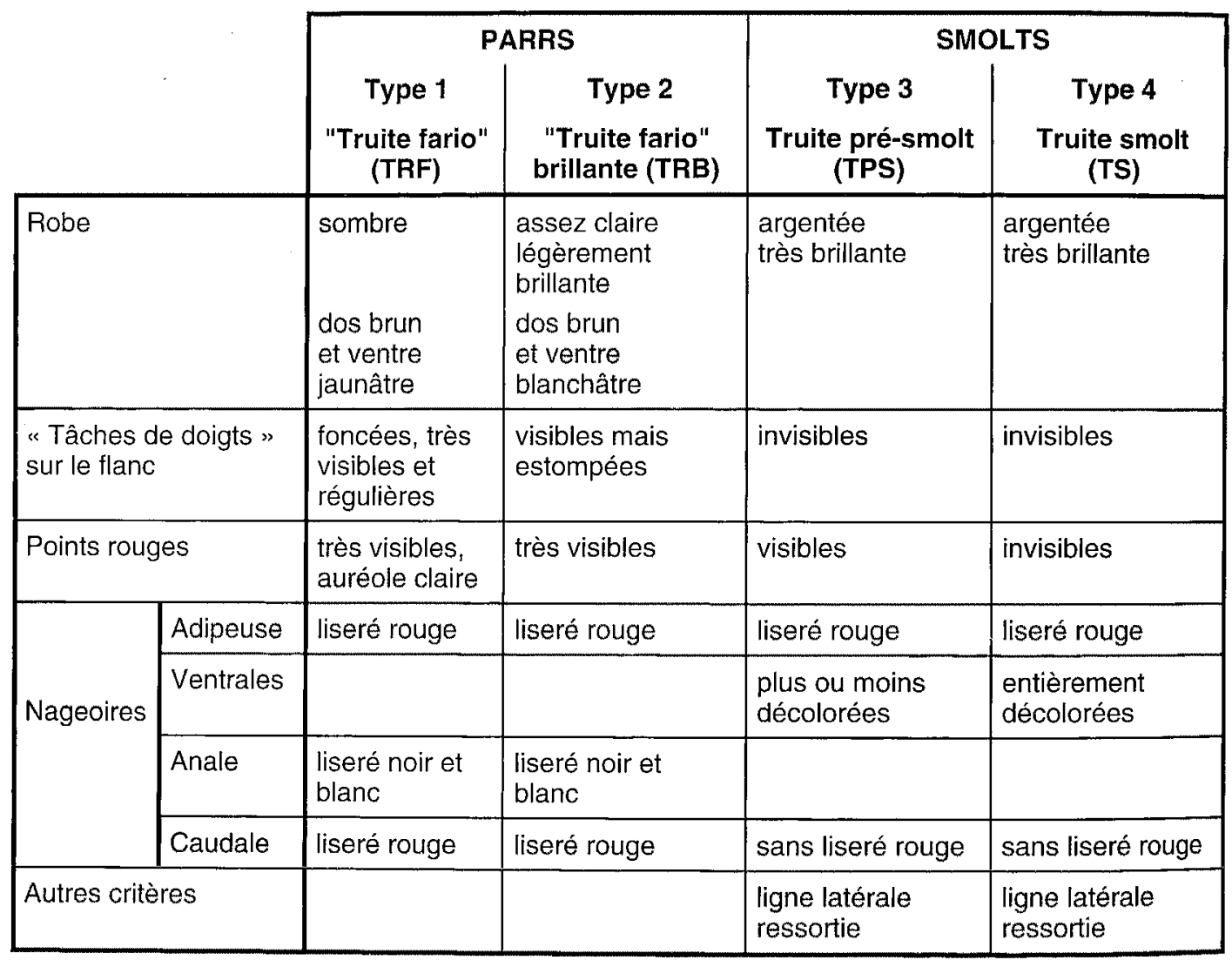

\section{RÉSULTATS-DISCUSSION}

\section{Stade adulte}

A ce stade, les deux formes se distinguent généralement bien à l'aide des deux critères retenus mais leur utilisation reste complémentaire. La truite de mer présente un gabarit nettement supérieur à celui de la forme rivière ainsi qu'une livrée blanche 
parsemée souvent de nombreuses taches noires en forme de croix de Saint-André qui peuvent se localiser même en dessous de la ligne latérale. Cependant, l'utilisation de ces critères n'apparaît pas suffisante pour distinguer les deux formes dans deux cas : (1) sur les grands fleuves où il existe des individus de grande taille de la forme rivière (70 cm sur le Rhin : OMBREDANE et al., (1998b) ; $80 \mathrm{~cm}$ et plus sur l'Allier et la Dordogne) ; (2) à une période proche de la reproduction qui voit la livrée blanche disparaître chez la forme marine et se confondre avec celle de la forme résidente. Ceci est confirmé par un pourcentage d'erreur non négligeable dans l'identification visuelle des deux formes par rapport à l'analyse scalimétrique (Tableau II). Par ailleurs, cette analyse montre que quel que soit le cours d'eau, l'identification visuelle a tendance à augmenter la proportion de truites de mer. En outre, même si l'examen de la structure des écailles donne des résultats très fiables, elle peut quelquefois rester insuffisante pour identifier les deux formes présentes sur les grands cours d'eau en raison de la très forte croissance des truites résidentes. Dans ce cas, le dosage du strontium dans les écailles permet de lever le doute (ROCHE, 1992). Cependant, l'utilisation d'une telle méthode présente quelques limites dans la mesure où le taux de strontium observé dépend du temps de séjour en eau douce des géniteurs lors de leur migration de reproduction (MAISSE et BAGLINIÈRE, 1990).

\section{Tableau II}

Pourcentage d'erreur dans l'identification visuelle entre la truite de rivière et celle de mer par rapport à l'analyse scalimétrique ; ( ) = nombre d'índividus.

\section{Table II}

Error percentage in visual identification between brown and sea trout compared to scale-reading $;()=$ number of fish.

\begin{tabular}{|c|c|c|c|c|c|}
\hline \multicolumn{7}{|c|}{ Pourcentage d'erreur } \\
\hline Cours d'eau & & Oir & Dordogne & Garonne & Rhin \\
\hline Montée estivale & moyenne & 3 & 23,7 & 14,7 & 12,3 \\
& & $(72)$ & $(80)$ & $(34)$ & $(139)$ \\
\cline { 2 - 6 } & extrêmes & $0-33,3$ & & & \\
\hline \multirow{3}{*}{ Montée automnale } & moyenne & 8 & & & \\
& & $(577)$ & & & \\
\cline { 2 - 6 } & extrêmes & $0-28,6$ & & & \\
\hline
\end{tabular}

\section{Stade juvénile}

Pour les poissons d'âge inférieur ou égal à 1+an, les critères retenus ne permettent pas de distinguer les deux formes. En fait, il ne semble exister actuellement aucune possibilité pour les distinguer morphologiquement au stade résident en place lors d'inventaires automnaux.

Lors de la migration printanière, les quatre phénotypes se distinguent par des aspects de la robe et des nageoires (Tableau 1) et par la valeur du coefficient de condition. Cette dernière diminue depuis le type $1(1,3)$ au type $4(0,9)$. Cependant, les différences ne sont significatives $(p<0,05)$ qu'entre le dernier et les trois autres (TANGUY, 1993).

Une distinction importante entre les quatre phénotypes réside dans les différences de niveau d'activité ATPasique. La valeur de cette dernière apparaît, en moyenne, significativement plus élevée $(p<0,01)$ chez les types 3 et 4 que chez les deux autres types (Figure 1A). Cela corrobore bien le fait qu'à l'instar du saumon, les juvéniles migrants de truite de mer smoltifient c'est-à-dire qu'ils présentent une augmentation 
significative de cette activité enzymatique (TANGUY et al., 1994). Par ailleurs, cette observation permet alors de considérer deux ensembles : celui des TPS et TS qui constitue le groupe de smolts et serait donc rattaché à la forme marine et celui des TRF et TRFB constituant celui des parrs et rattaché à la forme rivière. Néanmoins, la valeur de l'activité ATPasique ne peut pas être utilisée pour la discrimination des phénotypes à l'aide d'un abaque en raison de sa variabilité individuelle pour un type donné (Figure 1B). Cette variabilité des valeurs peut s'expliquer par trois raisons: (1) le faible niveau de précision des dosages de l'activité ATPasique, (2) l'absence de réelle coïncidence temporelle entre la migration, la mise en place de l'argenture et l'augmentation de l'ATPase, (3) l'appréciation visuelle relativement aléatoire du phénotype, particulièrement quand il s'agit de bien distinguer entre les types TRFB (type 2) et TPS (type 3).

A

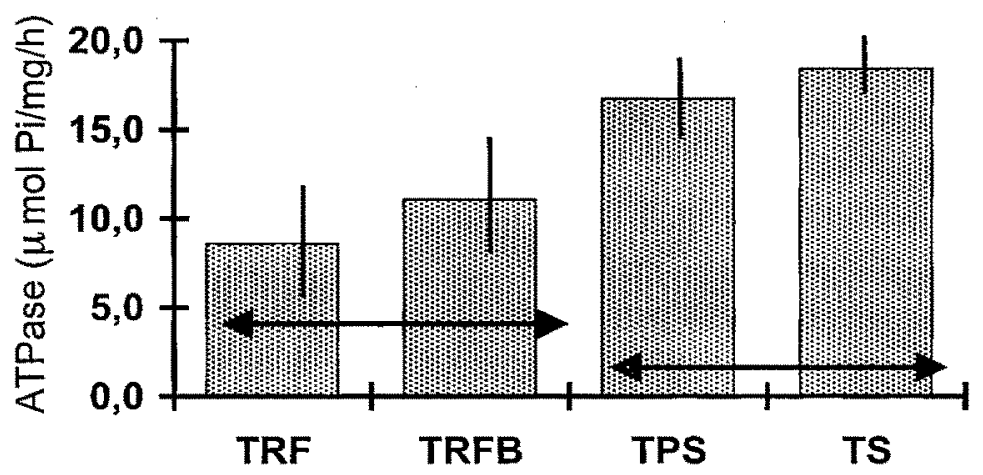

B

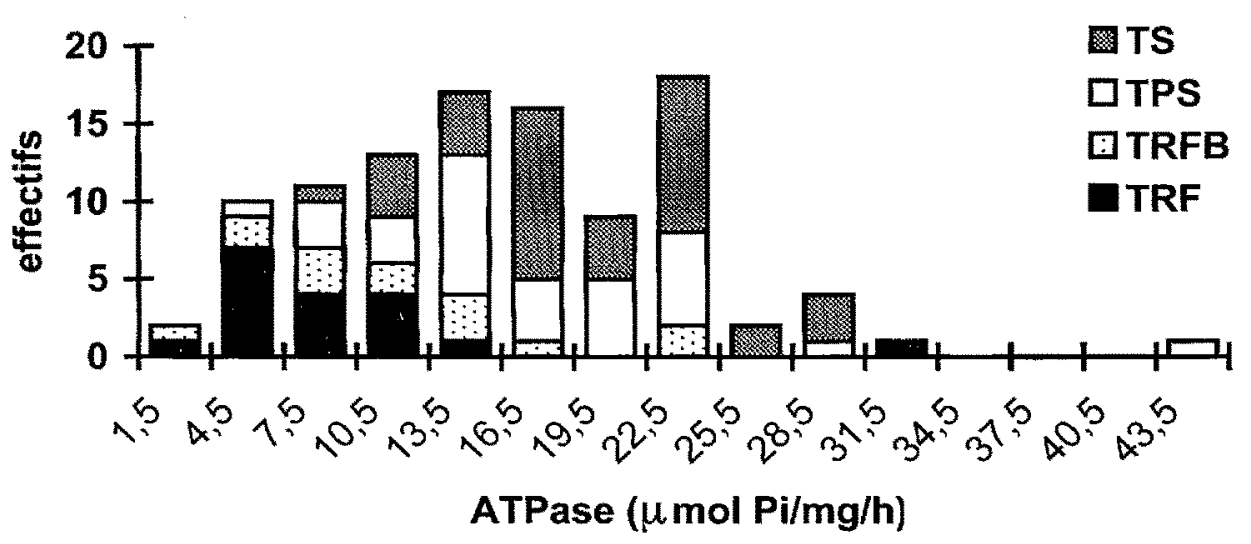

Figure 1

Variabilité de l'activité de la $\mathrm{Na}+/ \mathrm{K}+$ ATPase branchiale en fonction de leur phénotype chez les juvéniles migrants de truite du Douet de la Taille (Affluent de la Touques) - A : activités moyennes; les intervalles de confiance sont représentés par des traits verticaux (I) ; les flèches horizontales regroupent les moyennes statistiquement semblables (test $t, p<0,01$ ) - $B$ : répartition des valeurs individuelles dans des classes d'activité ATPasique de $3 \mu \mathrm{mol} \mathrm{Pi} / \mathrm{mg} / \mathrm{h}$.

\section{Figure 1}

Variability of gill $\mathrm{Na}+\mathrm{K}+\mathrm{APTase}$ activity in relation to phenotype in juveniles brown trout migrating in the Douet de la Taille brook (River Touques tributary) A : mean activities with confidence limits (I) ; horizontal arrows grouped together statistically equal means (test $t, p<0.01$ ) $-B$ : distribution of individual activity in $3 \mu \mathrm{mol} \mathrm{Pi} / \mathrm{mg} / \mathrm{h}$ ATPase activity class. 
Indépendamment de cette dernière observation, la distinction la plus nette entre les quatre phénotypes réside dans une augmentation du degré d'argenture de la robe lorsque l'on passe du type 1 (TRF) au type 4 (TS). Ainsi, pour s'affranchir de la variabilité de l'appréciation visuelle soulignée plus haut, les mesures colorimétriques de la robe ont été effectuées sur différentes parties du corps du poisson et montrent que la clarté augmente avec l'activité ATPasique et que la meilleure relation a été obtenue sur l'opercule (Figure 2). De la même manière, DUSTON (1995) en utilisant la clarté, montre des différences significatives du taux d'argenture entre les sites. Par ailleurs, il montre une mise en place progressive de l'argenture de la partie antérieure vers celle postérieure du poisson. En dépit de la variabilité des niveaux d'ATPase pour une valeur de clarté donnée, cette relation permet de valider la détermination visuelle des TS et TPS (groupe des smolts). La pertinence de cette détermination visuelle a pu être mise en évidence lors d'expériences de marquage-recapture. En effet, les individus TPS et TS déterminés visuellement et marqués ont donné au retour $90 \%$ d'adultes de truite de mer (OMBREDANE et al., 1996).

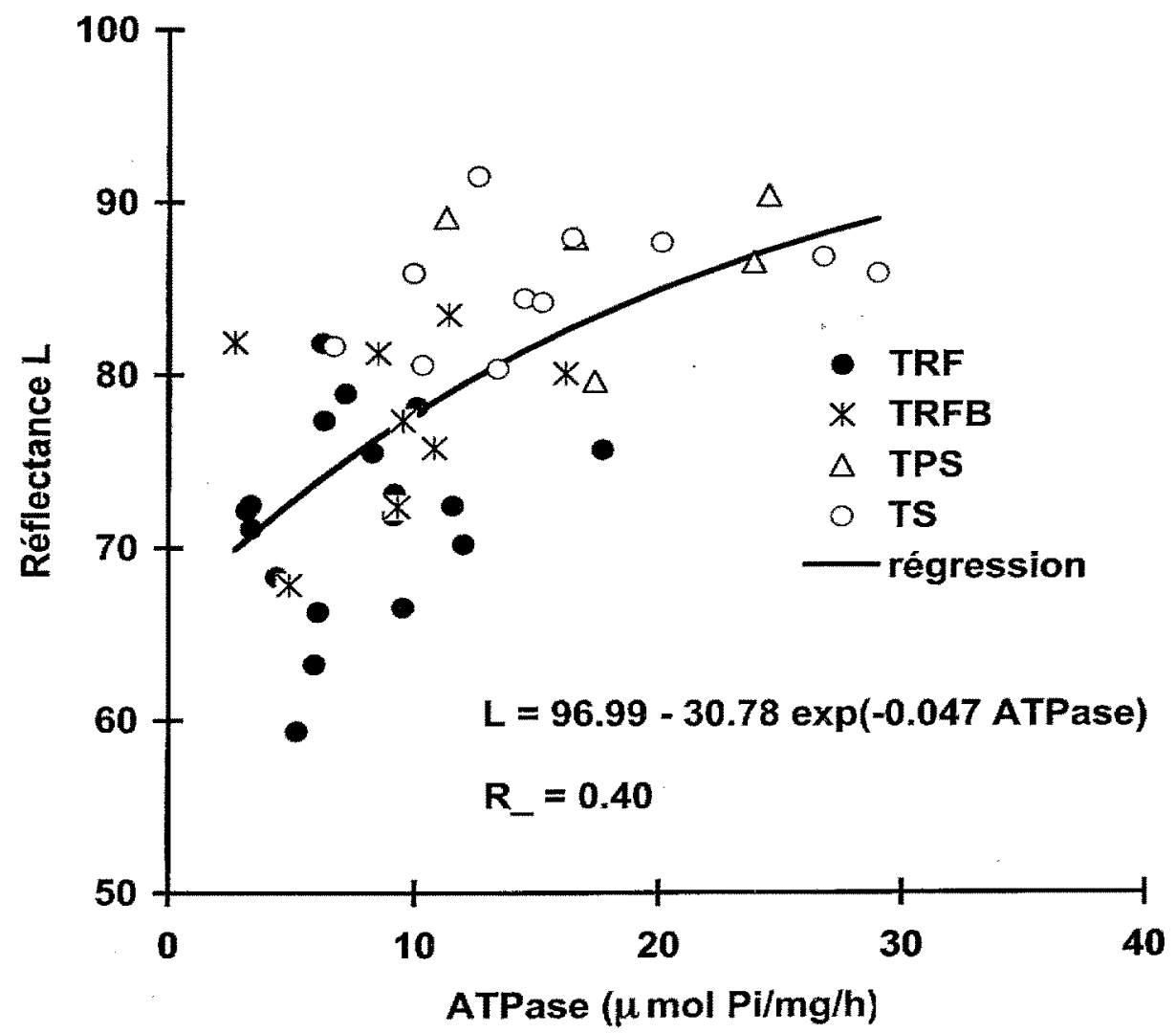

Figure 2

Relation asymptotique entre la clarté $L$ mesurée sur l'opercule et l'activité de la $\mathrm{Na}+\mathrm{K}+$ ATPase branchiale ( $\mu \mathrm{mol} \mathrm{Pi} / \mathrm{mg} / \mathrm{h}$ ) chez les quatre phénotypes de juvéniles de truite migrants sur l'Oir (affluent de la Sélune).

Figure 2

Asymptotic relationship between the lightness $L$ measured on the operculum and the gill $\mathrm{Na}+/ \mathrm{K}+$ ATPase activity ( $\mu \mathrm{mol} \mathrm{Pi} / \mathrm{mg} / \mathrm{h}$ ) in four phenotypes of juveniles brown trout migrating in River Oir (River Sélune tributary). 
D'autres études morphologiques utilisant la technique de l'analyse d'image ont également permis de séparer les smolts des truites résidentes (HAVEY et al., données non publiées) ; mais cette technique qui nécessite la mesure de 7 longueurs est plus difficile à mettre en oeuvre sur le terrain. Cependant, une utilisation des techniques photographiques pourrait être envisagée à condition de valider les mesures faites sur des individus prélevés et mesurés in situ.

\section{CONCLUSION ET PERSPECTIVES}

Actuellement, les critères de différentiation retenus entre les deux formes apparaissent performants au stade adulte à la condition d'y adjoindre un examen scalimétrique.

Il en est de même au stade juvénile migrant dans la mesure où les résultats obtenus ont confirmé l'identification visuelle du groupe des smolts. Néanmoins, une certaine incertitude demeure sur l'identification des différents phénotypes définis et sur la relation existant entre ces phénotypes et le devenir de l'individu. En effet, il a été montré que le juvénile de truite de rivière peut passer en mer sans smoltifier à partir d'une taille de $14 \mathrm{~cm}$ (TANGUY, 1993). Ainsi, des études complémentaires sont prévues afin de (1) définir le site optimal (flanc, nageoire pelvienne) permettant d'avoir le plus grand écart possible de clarté (L) ou d'un autre indice colorimétrique entre les phénotypes parr et smolt, (2) relier la mesure colorimétrique au devenir du poisson en tant qu'adulte. Dans ce dernier cas, des campagnes de marquage par pit-tag seront effectuées. Ce type de marquage individuel reste très performant car il n'induit aucune mortalité significative ni aucune modification du taux de croissance des poissons (OMBREDANE et al., 1998a).

Au stade juvénile en place ou résident, aucune distinction n'a pu être faite à partir des critères retenus. A l'avenir, il semble intéressant d'envisager une technique d'analyse d'image prenant en compte un nombre important de critères morphologiques comme ceux utilisés (37) par LASCAUX (1996) dans son étude de la variabilité morphologique de la truite commune adulte. La distinction à ce stade prend toute son importance lorsque l'on ne dispose d'aucun moyen pour échantillonner d'une manière correcte les juvéniles migrants et les adultes sur un cours d'eau.

Cependant sur les rivières où cet échantillonnage est possible, les critères proposés dans cette étude, permettent de distinguer les deux formes de truite et donc de suivre le niveau d'abondance des deux composantes d'un type de métapopulation qu'elles peuvent constituer. La prise en compte d'une telle structure apparaît nécessaire si l'on veut optimiser les efforts de protection et de conservation de l'espèce truite sur un bassin (COOPER et MANGEL, 1998).

\section{BIBLIOGRAPHIE}

BAGLINIĖRE J.L., 1991. La truite commune (Salmo trutta L.) : son origine, son aire de répartition, ses intérêts économique et scientifique. In : la truite : biologie et écologie, BAGLINIERE J.L. et MAISSE G. (Eds), INRA, Paris, 11-22.

BAGLINIERE J.L., MASSA F., MARCHAND F., 1997. Impact de l'intensification agricole sur l'état de la population de salmonidés d'un petit cours d'eau : le cas de l'Oir (Basse-Normandie). Rapport de synthèse, UMR INRA-ENSAR EQHC, Rennes, $14 \mathrm{p}$.

BAGLINIĖRE J.L., GUYOMARD R., HELAND M., OMBREDANE D., PRÉVOST E. (sous presse). Les cours d'eau à salmonidés : écologie des populations de poissons. In : l'eau et les organismes aquatiques, RIOU C., BONHOMME R., CHASSIN P., NEVEU A. et PAPY F. (Eds), INRA, Paris. 
COOPER A.B, MANGEL M., 1998. The dangers of ignoring metapopulation structure for the conservation of salmonids. Fish. Bull., 97, 213-226.

DAVAINE P., BEALL E., 1997. Introduction de salmonidés en milieu vierge (lles Kerguelen subantarctique) : enjeux, résultats, perspectives. Bull. Fr. Pêche Piscic., 344/345, 93-110.

DUSTON J., 1995. A light-reflectance meter to quantify silvering during smolting in Atlantic salmon. J. Fish Biol., 46, 912-914.

EÜZENAT G., FOURNEL F., FAGARD J.L., 1991. La truite de mer (Salmo trutta L.) en Normandie/Picardie. In : la truite : biologie et écologie, BAGLINIËRE J.L. et MAISSE G. (Eds), INRA, Paris, 183-213.

GUYOMARD $R_{n}, 1991$. Diversité génétique et gestion des populations naturelles de truite commune. In : la truite, biologie et écologie, BAGLINIËRE J.L. et MAISSE G. (Eds), INRA, Paris, 215-235.

LASCAUX J.M., 1996. Analyse de la variabilité morphologique de la truite commune (Salmo trutta) dans les cours d'eau du bassin pyrénéen méditerranéen. Thèse Doctorat, INPT Toulouse, $108 \mathrm{p}$.

MAISSE G., BAGLINIĖRE J.L., 1990. Limites de la méthode de reconnaissance de l'origine (marine ou dulcicole) de la truite (Salmo trutta L.) au moyen de l'analyse de la teneur en strontium des écailles. Bull. Fr. Pêche Piscic., 316, 24-27.

MAISSE G., BAGLINIĖRE J.L., 1991. Biologie de la truite commune (Salmo trutta L.) dans les rivières françaises. In : la truite : biologie et écologie, BAGLINIERE J.L. et MAISSE G. (Eds), INRA, Paris, 25-45.

NÄSLUND I., 1993. Migratory behaviour of brown trout (Salmo trutta) : importance of genetic and environmental factors. Ecol. Freshw. Fish., 2, 51-57.

OMBREDANE D., 2000. Prévision du stade smolt par des mesures colorimétriques de l'argenture chez le saumon atlantique (Salmo salar). Rapp., UMR INRA-ENSAR EQHC, Rennes, $33 \mathrm{p}$.

OMBREDANE D., SIEGLER L., BAGLINIERE J.L., PRUNET P., 1996. Migration et smoltification des juvéniles de truite (Salmo trutta) dans les cours d'eau de BasseNormandie. Cybium, 20 (suppl.), 27-42.

OMBREDANE D., BAGLINIERE J.L., MARCHAND F., 1998a. The effects of passive integrated transponder tags on survival and growth of juvenile brown trout (Salmo trutta) and their use for studying movements in a small river. Hydrobiologia, 371/372, 99-106.

OMBREDANE D., ROCHE P., BAGLINIERE J.L., EDEL G., GERLIER M., GIPPET B., 1998b. Estimation des caractéristiques biologiques des truites de mer adultes (Salmo trutta) du Rhin supérieur. Bull. Fr. Pêche Piscic., 350/351, 655-673.

RICHARD A., BAGLINIERE J.L., 1990. Description et interprétation des écailles de truite de mer (Salmo trutta L.) des deux rivières de Basse-Normandie : l'Orne et la Touques. Bull. Fr. Pêche Piscic., 319, 239-257.

ROCHE P., 1992. Mise en évidence de l'écotype truite de mer dans ies captures de grandes truites (Salmo trutta L.) du Rhin supérieur. Bull. Fr. Pêche Piscic., 324, 36-44.

TANGUY J.M., 1993. La smoltification de la truite de mer (Salmo trutta) : caractérisation éco-physiologique des juvéniles en milieu contrôle et en milieu naturel. Thèse Doc. Ing., Ecole Nationale Supérieure Agronomique de Rennes, Produc. Anim., option halieutique, $106 \mathrm{p}$.

TANGUY J.M., OMBREDANE D., BAGLINIERE J.L., PRUNET P., 1994. Aspects of parr smolt transformation in anadromous and resident forms of brown trout (Salmo trutta) in comparison with Atlantic salmon (Salmo salar). Aquaculture, 121, 51-63. 